Bull. Mater. Sci., Vol. 8, No. 4, October 1986, pp. 467-470.

(C) Printed in India.

\title{
Growth of low dislocation density single crystals of nickel
}

\author{
S K KHANNA and K GOVINDA RAJAN \\ Materials Science Laboratory, Indira Gandhi Centre for Atomic Research, Kalpakkam \\ 603102 , India
}

MS received 16 January 1986; revised 24 April 1986

\begin{abstract}
Low dislocation density single crystals of nickel have been grown at high ambient pressure by the Czochralski method. X-ray Laue picture shows that the crystals are strain-free. The dislocation density was determined to be $<10^{3} / \mathrm{cm}^{2}$ by the etching procedure. It was found that the necking and cone regions are very critical in the dislocation introduction in the crystals. An increase in the ambient pressure used during the growth seems to aid the crystal quality.
\end{abstract}

Keywords. Nickel crystals; high pressure growth; Czochralski technique.

\section{Introduction}

The physical properties of the crystalline materials are sensitive to the presence of lattice imperfections such as dislocations which may be present in the as-grown crystals. A significant contribution was made by Dash (1958) who discovered the method of eliminating dislocations in the melt-grown crystals. A thin neck is grown on top of the growing crystal by employing large pulling rates. The line defects in the neck grow out to the nearby surface. Moreover, only a very small number of dislocation lines are allowed from the seed crystal. Dash's technique is particularly suitable for growing nearly perfect single crystals of metals where the necking procedure will ensure a sufficiently small temperature gradient in the main body of the crystal. We have employed this procedure to grow low dislocation density single crystals of nickel. The other objective of the present work has been to study the influence of higher ambient pressure on the quality of the crystal.

\section{Experimental details}

We have employed the Czochralski technique for the growth of single crystals where the crystal cools in an unconstrained manner thus making it possible to produce low dislocation density crystals. Moreover, the necking procedure can be employed, and the shape of the growing crystal can be controlled to a considerable extent. The details of the main equipment used for the Czochralski growth have been described elsewhere (Khanna et al 1986). The initial charge in the form of pellets $(99.99 \%$ pure nickel) was placed in an alumina crucible which was completely supported by a graphite container. A $99.99 \%$ pure nickel wire was used for the seed. The system was filled with high purity argon to a pressure of 6 bars. The seed crystal was carefully wetted in the melt before initiating the crystal pulling. The seed crystal and the crucible were rotated in the opposite direction at $10 \mathrm{rpm}$. This was done to intermix the molten mass and to ensure a constant circumferential temperature. A thin neck of $0.4 \mathrm{~mm}$ was grown on the top of the crystal. The diameter was controlled only by 
continuously monitoring the melt temperature, and the pulling speed during the growth was maintained at a constant level of $0.2 \mathrm{~mm} / \mathrm{min}$. This way it was ensured that there were no sudden mechanical shocks given to the solid-liquid interface. The pulling speed $v_{p}$ does not coincide with the growth rate $V$. As the crystal grows, the melt level lowers. The two rates are related to each other by the following relation (Paorici 1982)

$$
V=\frac{V_{p}}{1-\left[\left(\rho_{s} / \rho_{L}\right)\left(d_{s} / d_{L}\right)^{2}\right]}
$$

where $d_{s}, d_{L}$ are diameters of the growing crystal and the melt in the crucible, $\rho_{s}$ and $\rho_{L}$ are the densities of nickel in solid and liquid phases. The crucible lift rate was fixed at $0.02 \mathrm{~mm} / \mathrm{min}$. On completion of the growth the crystal was cooled down very slowly at $10^{\circ} \mathrm{C} / \mathrm{min}$ to avoid dislocation generation due to thermal gradisints.

\section{Results and discussion}

By using high pressure Czochralski technique we have grown single crystals of nickel ( $6 \mathrm{~mm}$ diameter and $25 \mathrm{~mm}$ long). Single crystallinity was examined by exposing the crystal to the $x$-ray beam and recording the Laue back reflection picture. Figure 1 shows that the crystal is both a single crystal and strain-free. The nickel crystals reported so far (Hayashi et al 1974; Kuriyama et al 1977) have been grown from melt either in vacuum or in an inert gas environment maintained at $1 \mathrm{~atm}$. pressure. The dislocation density reported in these crystals was fairly high (Hayashi et al 1974). In order to find out the dislocation density of the present high pressure Czochralski grown crystals, these were cut normal to the growth axis and then characterized by metallographic techniques. The crystals were mechanically polished by using diamond paste down to a $3 \mu \mathrm{m}$ surface finish and etched in a solution of $\mathrm{HNO}_{3}$ and acetic acid (Brandes 1983). The dislocation density was measured by first identifying those etch pits which were due to dislocations. For this the crystal surface was successively etched and the continuity of etch pits was followed. Etching resulted in well-defined etch pits which were triangular in shape (figure 2) and which persisted even after the removal of successive layers from the crystal surface. Slight roughness of the etched surface, seen in the photograph, could be due to the effects of mechanical polishing. We have used only upto $3 \mu \mathrm{m}$ grain size diamond paste. This procedure yielded an estimate of $<10^{3}$ dislocations $/ \mathrm{cm}^{2}$, indicating that the crystal grown in an argon gas atmosphere at a pressure slightly higher than the ambient is of good quality. Low dislocation density was obtained by eliminating the dislocations from the seed crystal in the neck, and the dislocations can be reintroduced if the diameter of the cone region is not controlled. The growth of the cone region was very important for obtaining low dislocation density single crystals of nickel. The cone region was grown such that the top angle was small. The cone could thus grow out with minimum dislocations. On the other hand, with large top angles, the internal stresses in the growing crystal would have been high and that would cause dislocation generation in the cone region. With dislocations being kept at minimum level in the cone region, further growth of the crystal was accomplished such that the crystal diameter stayed more or less constant. With this the dislocation multiplication in the lower part of the crystal was prevented to a great extent. 

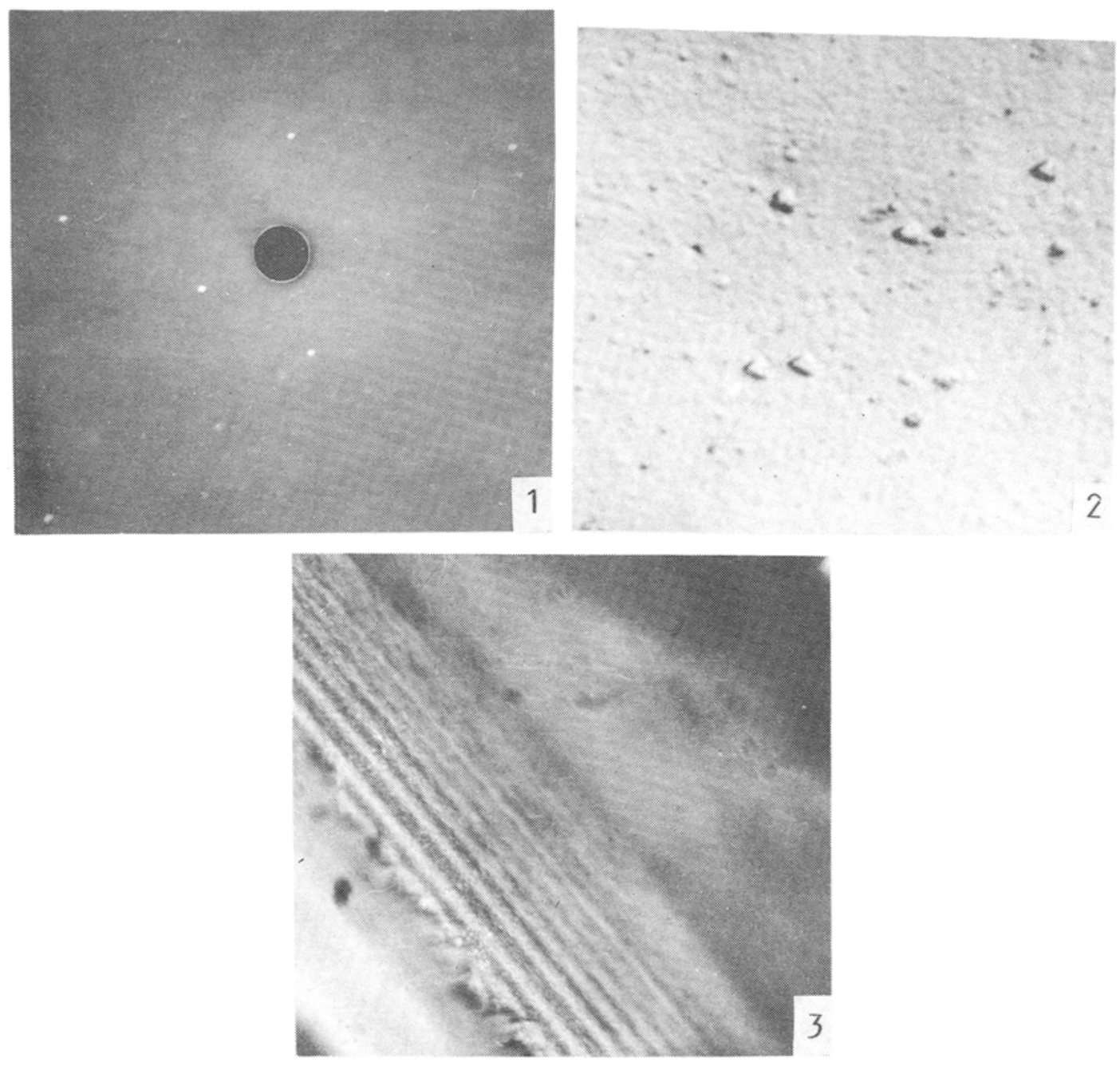

Figures 1-3. 1. X-ray back reflection picture of single crystal of nickel. 2. Dislocation etch pits in nickel crystal. 3. Radial growth striations in nickel single crystal.

Striations observed normal to the growth axis $<111>$ (figure 3 ) showed the shape of the solid-liquid interface. No axial growth striations were observed. Since the crystals were not doped with any other material, these striations are not due to the solute variations. These were found to be not periodic over the length of the crystal and were observed at an extremely small number of places. The unintentional dopants that might exist in $99.99 \%$ pure nickel will, on the other hand, give rise to striations which will be periodic over the entire length of the crystal. Also no discoloration of the alumina crucible material was observed after the growth experiments, implying that there was no reaction of nickel with $\mathrm{Al}_{2} \mathrm{O}_{3}$ crucible. The spacing between the two adjoining striations was measured to be $2-3 \mu \mathrm{m}$. These striations can be attributed to the possible thermal fluctuations present across the interface due to the thermal convection in the melt. This is because the temperatures 
and temperature gradients commonly used in the growth of single crystals of nickel are not very low to eliminate thermal convection. A spacing of the above mentioned magnitude is typical of such fluctuations. Although low temperature gradients in the crystal were favourable for the low dislocation density growth, with such low temperature gradients in the melt, the melt convection reduces making the heat transfer from the crucible wall to the crystal interface small. Subsequently it became very difficult to control the crystal diameter.

Low dislocation density $\left(<10^{3}\right.$ dislocations $\left./ \mathrm{cm}^{2}\right)$ observed in nickel single crystals grown in argon gas at 6 bar pressure indicates that the high ambient pressure does not reduce the quality of the crystal. On the other hand, the crystal is mechanically extremely soft at its meiting terrperature and a slight ambient pressure perhaps prevents dislocation generation by reducing the thermal stresses at the interface. Compared to the vacuum-grown crystals these crystals have lower dislocation density and their microstructure does not show any grain boundaries.

\section{Conclusions}

Strain-free single crystals of nickel have been grown at 6 bar inert gas pressure. The slight hydrostatic pressure environment and the necking are found to improve the crystal quality. The dislocation density of these crystals was determined to be less than $10^{3}$ dislocations $/ \mathrm{cm}^{2}$.

\section{Acknowledgement}

The authors are thankful to Shri $M$ Sekar for assistance in the experiments and Shri G V N Rao for help in x-ray work.

\section{References}

Brandes E A 1983 Smithells metals reference book (London: Butterworths) p. 10

Dash W C 1958 J. Appl. Phys. 29736

Hayashi S, Echigoya J, Hariu H, Sato T, Nakamichi T and Yamamoto M 1974 J. Cryst. Growth 24/25 $422-425$

Khanna S K, Sekar M, Michael David A, Govinda Rajan K and Bhaskar Rao P 1986 Pramana (J. Phys.) 26151

Kuriyama M, Boettinger M J and Burdette H E 1977 Advances in x-ray analysis (New York: Plenum Press) (eds) H F McMuradie, C S Barrett, J B Newkirk and C O Rund Vol. 20, p. 245

Pamplin B R 1975 Crystal growth (New York: Pergamon Press)

Paorici C 1982 Proc. international school on synthesis, crystal growth and characterization (ed) K Lal (Amsterdam: North-Holland) p. 170 